\title{
BMJ Open Multicentre, randomised, placebo- controlled trial of extract of Japanese herbal medicine Daikenchuto to prevent bowel dysfunction after adult liver transplantation (DKB 14 Study)
}

\author{
Toshimi Kaido, ${ }^{1}$ Tsuyoshi Shimamura, ${ }^{2}$ Yasuhiko Sugawara, ${ }^{3}$ Hiroshi Sadamori, ${ }^{4}$ \\ Ken Shirabe, ${ }^{5}$ Michio Yamamoto, ${ }^{6}$ Shinji Uemoto ${ }^{1}$
}

To cite: Kaido T,

Shimamura T, Sugawara $Y$, et al. Multicentre, randomised, placebocontrolled trial of extract of Japanese herbal medicine Daikenchuto to prevent bowel dysfunction after adult liver transplantation (DKB 14 Study). BMJ Open 2015;5: e008356. doi:10.1136/ bmjopen-2015-008356

- Prepublication history for this paper is available online. To view these files please visit the journal online (http://dx.doi.org/10.1136/ bmjopen-2015-008356).

Received 31 March 2015 Revised 26 June 2015 Accepted 7 August 2015

CrossMark

For numbered affiliations see end of article.

Correspondence to Professor Toshimi Kaido; kaido@kuhp.kyoto-u.ac.jp

\section{ABSTRACT}

Introduction: This multicentre randomised controlled clinical trial will aim to determine the ability of an extract (TJ-100) of Daikenchuto (traditional Japanese herbal medicine; Kampo) to prevent bowel dysfunction in at least 110 patients after liver transplantation (LT).

Methods and analysis: The following co-primary end points will be evaluated on postoperative day 7 : total oral and enteral caloric intake, abdominal distension and abdominal pain. The secondary end points will comprise sequential changes of total oral and enteral caloric intake after $\mathrm{LT}$, sequential changes in numeric rating scales for abdominal distension and pain, elapsed time to the first postoperative passage of stool, quality of life assessment using the Gastrointestinal Symptom Rating Scale score (Japanese version), postoperative liver function, liver regeneration rate, incidence of bacteraemia and bacterial strain, trough level of immunosuppressants, occurrence of acute cellular rejection, discharge or not within 2 months after LT, sequential changes of portal venous flow to the graft and ascites discharge. The two arms of the study will comprise 55 patients per arm. Ethics and dissemination: The study has been conducted according to the CONSORT statement. All participants signed a written consent form, and the study has been approved by the institutional review board of each participating institute and conducted in accordance with the Declaration of Helsinki of 1996. The findings will be disseminated through scientific and professional conferences, and in peer-reviewed journals.

Trial registration number: The DKB 14 Study was registered in the University Hospital Medical Information Network Clinical Trial Registration (UMINCTR), Japan (registration number: UMIN000014326) during 2014.

\section{INTRODUCTION}

Liver transplantation (LT) is one of the most extensive surgeries for patients with end- stage liver diseases including liver cirrhosis, hepatocellular carcinoma and acute liver failure. Moreover, protein-energy malnutrition is common in patients with end-stage liver disease requiring LT and closely associated with post-transplant risk of morbidity and mortality. ${ }^{1-3}$ Such patients are usually accompanied by bowel dysfunction after LT due to long surgical durations and wide abdominal incisions, which prevents early postoperative food intake either orally or via an enteral tube and subsequently leads to worsening malnutrition.

The enhanced recovery after surgery (ERAS) protocol has recently been introduced to various types of surgery including organ transplantation. ${ }^{4-7}$ Postoperative management recommended by the ERAS protocol includes the early initiation of normal food intake or enteral feeding after gastrointestinal surgery. Moreover, early tube feeding (within $24 \mathrm{~h}$ ) is indicated for patients in whom early oral nutrition cannot be initiated due to obvious undernutrition at the time of surgery. However, normal food intake or enteral feeding cannot be started when a patient has bowel dysfunction.

Daikenchuto is a traditional Japanese herbal medicine (Kampo) that has been frequently prescribed to prevent and treat postoperative ileus in Japan. ${ }^{8} 9$ The powdered extract of Daikenchuto, TJ-100 (Tsumura \& Co, Tokyo, Japan), is available as an aqueous extract containing 2.2\% Japanese pepper, $5.6 \%$ processed ginger, $3.3 \%$ ginseng and $88.9 \%$ powdered maltose syrup. A recent multicentre phase III trial (JFMC40-1001) found that TJ-100 significantly reduced the elapsed time to the first bowel movement compared with a placebo after hepatic 
resection. ${ }^{10}$ However, the ability of TJ-100 to normalise bowel paralysis after LT and its effects on early total caloric intake delivered orally or enterally after surgery (a key element of the ERAS protocol) have not been prospectively investigated. Therefore, we designed a multicentre, randomised, double-blind, placebo-controlled trial to determine the enhancement effects of TJ-100 on oral and enteral caloric intake and its ability to prevent postoperative paralytic ileus in patients after undergoing living donor LT (LDLT) or deceased donor LT (DDLT).

\section{METHODS AND ANALYSIS \\ Objective}

Postoperative early oral or enteral intake is a crucial element of the ERAS protocol. The DKB 14 Study is a multicentre randomised, double-blind, placebo-controlled trial that aims to determine the enhancement effect of $\mathrm{TJ}-100$ on oral and enteral caloric intake and its ability to prevent postoperative paralytic ileus in patients after LDLT and DDLT.

\section{Resources}

A research grant from Tsumura \& Co, Tokyo, Japan.

\section{End points}

The following primary end points will be evaluated at postoperative day (POD) 7:

- Total oral or enteral caloric intake;

- Abdominal distension determined using numeric rating scales (NRS);

- Abdominal pain determined using NRS.

The following secondary end points will be evaluated:

- Chronological changes in total oral or enteral caloric intake at POD 3, 5, 7, 10 and 14;

- Chronological changes in NRS at POD 5, 7, 10 and 14

- Elapsed time from extubation until the first postoperative defaecation;

- Quality of life assessment using the Gastrointestinal Symptom Rating Scale score (Japanese V.1.2) at POD 7 and 14 ;

- Liver function determined by measuring total protein, albumin, total bilirubin, direct bilirubin, aspartate aminotransferase, alanine aminotransferase, $\gamma$-glutamyl transpeptidase, choline esterase at POD 3, $5,7,10$ and 14 ;

- Liver regeneration rates determined by CT and calculated as postoperative liver volume/graft liver weight at the time of LT between POD 14 and 21 and graft volume for all patients except those who underwent whole LT;

- The incidence of bacteraemia until POD 14 and bacterial strain;

- Trough levels of immunosuppressants at POD 3, 5, 7, 10 and 14 ;

- The incidence of acute cellular rejection until POD 14;
- Discharge or not within 2 months after LT;

- Flow speed and volume of the portal vein at POD 3, $5,7,10$ and 14 ;

- Ascites volume (mL/day) from abdominal drain at POD 3, 5, 7, 10 and 14 .

\section{Eligibility criteria}

Inclusion

- Patients with end-stage liver disease who are scheduled to undergo LT

- Age $\geq 20$ years at the time of registration

- Satisfies the indication criteria for LT at each participating institution

- Written informed consent provided to participate in the study

\section{Exclusion}

- Uncontrollable active infection other than liver

- Uncontrollable malignant diseases other than hepatocellular carcinoma

- Clinically problematic dysfunction of other organs

- Likely to have severe intra-abdominal adhesion due to a history of surgeries or past history of mechanical ileus

- Medication with antipsychotic, antidepressant or gastrointestinal prokinetic drugs

- Patients who take other Kampo medicines

- Women who are pregnant or lactating

- Any other medical condition that would render a patient unsuitable for inclusion according to the opinion of the investigator (at each institution).

\section{Registration}

An eligibility report form will be delivered to the registration centre at the Institute for Advancement of Clinical and Translational Science, Kyoto University Hospital. Eligible patients will be centrally randomised to either Arm A (TJ-100) or Arm B (placebo) in accordance with the minimisation method for assigning patients to Arm A or Arm B according to the type of LT (LDLT or DDLT), body weight $(<60$ or $\geq 60 \mathrm{~kg}$ ), age ( $<50$ or $\geq 50$ years) and the institution as variables before LT. Information regarding required follow-up evaluations will then be sent from the registration centre at the Institute for Advancement of Clinical and Translational Science.

The randomisation list will not be known in advance by the investigators. The statistical analysis and preparation of tables and graphs for the report of the study by the statistician of the study will be blinded to the extent possible. The unblinding may take place only after all data have been entered into the database of the study, all requests have been closed and the database has been frozen by the Data Manager of the study.

Treatment methods

Arm A: TJ-100 group

TJ-100 (5 $\mathrm{g}$ in solution) will be administered either orally or enterally via a tube three times per day 
immediately before meals or every $8 \mathrm{~h}$ for 14 consecutive days between POD 1 and 14 .

\section{Arm B: placebo group}

Placebo ( $5 \mathrm{~g}$ in solution) will be administered either orally or enterally via a tube three times per day immediately before meals or every $8 \mathrm{~h}$ for 14 consecutive days between POD 1 and 14.

TJ-100 and a matching placebo were manufactured by Tsumura \& Co (Tokyo, Japan).

We monitor adherence by hearing each patient and checking the number of trial drugs (TJ-100 or placebo) that are not administered.

\section{Prohibited or permitted drugs}

Prohibited drugs: drugs that are known to promote bowel movement are prohibited during the protocol treatment: erythromycin, acetylcholine chloride, itopride hydrochloride, mosapride citrate, aclatonium napadisilate, neostigmine methylsulfate, pantethine, panthenol, prostaglandin $\mathrm{F} 2 \alpha$, prosultiamine, fursultiamine, trimebutine maleate, amidotrizoate sodium meglumine.

Permitted drugs: metoclopramide or domperidone to treat postoperative nausea/vomiting, and immunosuppressants including tacrolimus, cyclosporine, steroid, azathioprine, mizoribin and mycophenolate mofetil.

\section{Criteria for discontinuing the protocol treatment}

Grade 3 postoperative diarrhoea or other clinical adverse effects (CTCAE V.4.0 criteria).

\section{Data collection}

Prospective data about all patients including medical history, physical findings, laboratory findings, perioperative clinical information and complications will be collected.

\section{Study design and statistical analysis}

The primary end points will be statistically evaluated at POD 7 (total oral or enteral caloric intake, abdominal distension and abdominal pain determined using NRS).

The multiplicity issue (inflation of type I error) due to the analysis of three end points will be addressed using the fixed-sequence testing method. The order of the tests will be fixed, which controls the familywise error rate. The testing procedure will start from a hypothesis about the total oral and enteral caloric intake, and will be followed by the NRS for abdominal distension and the NRS for abdominal symptoms in that order. Each test will proceed only if all previously tested hypotheses have been rejected. Each hypothesis will be tested at the $5 \%$ significance level. The sample size was calculated on the basis of our previous finding that the mean value of the total oral and enteral caloric intake at POD 7 without the administration of TJ-100 is about $1000 \mathrm{kcal} /$ day $(\mathrm{SD}=850)$ (unpublished data). We speculated that patients administered with TJ-100 would have an intake of $500 \mathrm{kcal} /$ day more than those who were not administered with TJ-100. Thus, assuming that the two arms have the same $\mathrm{SD}$, the sample size was calculated on the basis of a t test with a two-sided significance level of $5 \%$ and a power of $80 \%$, which resulted in a requirement of 47 patients per group. Taking about $15 \%$ exclusion from analysis into account, the number of patients to be accrued was set at 55 per treatment arm (110 in total). If the sample for analyses is 47 per group and the twosided significance level is $5 \%$, and if the probability of an event in which the value of the NRS in Arm A is larger than that in Arm B is about 0.62, then the Wilcoxon rank-sum test would have an approximate power of $80 \%$ for each NRS test of abdominal distension and abdominal symptoms. The first primary end point, the total oral and enteral caloric intake at POD 7, will be compared between the two treatment groups using the $\mathrm{t}$ test. The second and the third primary end points, the values of NRS on abdominal distension and abdominal pain, will be compared between the two treatment groups using the Wilcoxon rank-sum test. When the total oral and caloric enteral intake data at POD 7 for 50 patients are collected, we will conduct an interim analysis to confirm the distribution of total oral and enteral caloric intake at POD 7 under double-blind conditions. If the distribution obviously deviates from the normal distribution, then the Wilcoxon rank-sum test will be used for the primary analysis of the total oral and enteral caloric intake at POD 7.

\section{Participating institutions}

Fourteen leading Japanese institutions that perform LT will participate in this trial.

\section{Registration of the protocol}

The protocol was approved by the institutional review board of each participating institute and conducted in accordance with the Declaration of Helsinki of 1996. Written informed consent will be obtained from all patients before enrolment and randomisation by investigators. The DKB 14 Study was registered in the University Hospital Medical Information Network Clinical Trial Registration (UMIN-CTR), Japan (registration number: UMIN000014326) during 2014.

\section{Study status}

This study is currently collecting data and there has not been any publication concerning the analysis of the data collected to date.

\section{Author affiliations}

${ }^{1}$ Division of Hepato-Biliary-Pancreatic and Transplant Surgery, Department of Surgery, Graduate School of Medicine, Kyoto University, Kyoto, Japan 2Division of Organ Transplantation, Hokkaido University Hospital, Sapporo, Japan

${ }^{3}$ Artificial Organ and Transplantation Division, Department of Surgery, Graduate School of Medicine, The University of Tokyo, Tokyo, Japan ${ }^{4}$ Department of Gastroenterological Surgery, Okayama University Graduate School of Medicine, Dentistry and Pharmaceutical Sciences, Okayama, Japan ${ }^{5}$ Department of Surgery and Science, Graduate School of Medical Sciences, Kyushu University, Fukuoka, Japan 
${ }^{6}$ Institute for Advancement of Clinical and Translational Science, Kyoto University Hospital, Kyoto, Japan

Contributors TK and SU conceived the study. All the authors were involved in development of the study protocol. TK prepared the first draft of the manuscript and all the authors critically reviewed, revised and approved the subsequent and final version.

Funding This work was supported by a research grant from Tsumura \& Co, Tokyo, Japan.

Competing interests None declared.

Ethics approval The protocol was approved by the institutional review board of each participating hospital.

Patient consent Obtained.

Provenance and peer review Not commissioned; externally peer reviewed.

Open Access This is an Open Access article distributed in accordance with the Creative Commons Attribution Non Commercial (CC BY-NC 4.0) license, which permits others to distribute, remix, adapt, build upon this work noncommercially, and license their derivative works on different terms, provided the original work is properly cited and the use is non-commercial. See: http:// creativecommons.org/licenses/by-nc/4.0/

\section{REFERENCES}

1. Selberg O, Böttcher J, Tusch G, et al. Identification of high-and low-risk patients before liver transplantation: a prospective cohort study of nutritional and metabolic parameters in 150 patients. Hepatology 1997;25:625-57.
2. Sanchez AJ, Aranda-Michel J. Nutrition for the liver transplant patient. Liver Transplant 2006;12:1310-16.

3. Stickel F, Inderbitzin D, Candinas D. Role of nutrition in liver transplantation for end-stage chronic liver disease. Nutr Rev 2008:66:47-54.

4. Weimann A, Braga M, Harsanyi L, et al. ESPEN Guidelines on Enteral Nutrition: Surgery including organ transplantation. Clin Nutr 2006;25:224-44.

5. Lassen K, Coolsen MM, Slim K, et al., ERAS $®$ Society; European Society for Clinical Nutrition and Metabolism; International Association for Surgical Metabolism and Nutrition. Guidelines for perioperative care for pancreaticoduodenectomy: Enhanced Recovery After Surgery (ERAS $®$ ) Society recommendations. Clin Nutr 2012;31:817-30

6. Gustafsson UO, Scott MJ, Schwenk W, et al., Enhanced Recovery After Surgery Society. Guidelines for perioperative care in elective colonic surgery: Enhanced Recovery After Surgery (ERAS $®$ ) Society recommendations. Clin Nutr 2012;31:783-800.

7. Mortensen K, Nilsson M, Slim K, et al., Enhanced Recovery After Surgery (ERAS $\AA$ ) Group. Consensus guidelines for enhanced recovery after gastrectomy: Enhanced Recovery After Surgery (ERAS®) Society recommendations. Br J Surg 2014;101:1209-29.

8. Endo S, Nishida T, Nishikawa K, et al. Dai-kenchu-to, a Chinese herbal medicine, improves stasis of patients with total gastrectomy and jejunal pouch interposition. Am J Surg 2006;192: 9-13.

9. Iwai $\mathrm{N}$, Kume $\mathrm{Y}$, Kimura $\mathrm{O}$, et al. Effects of herbal medicine Dai-Kenchu-to on anorectal function in children with severe constipation. Eur J Pediatr Surg 2007:17:115-18.

10. Shimada M, Morine $\mathrm{Y}, \mathrm{Nagano} \mathrm{H}$, et al. Effect of TU-100, a traditional Japanese medicine, administered after hepatic resection in patients with liver cancer: a multi-center, phase III trial (JFMC40-1001). Int J Clin Oncol 2015;20:95-104. 